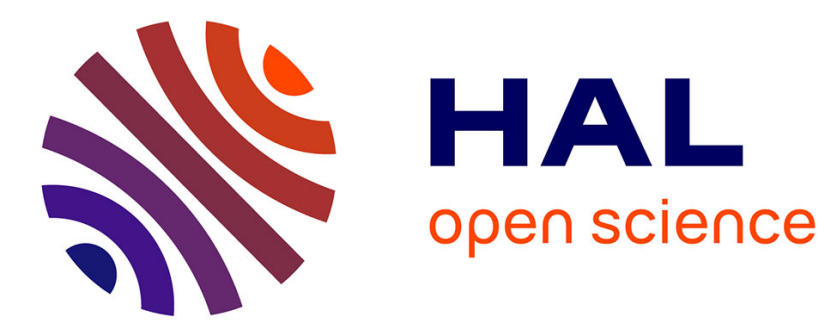

\title{
Brain-inspired computational paradigm dedicated to fault diagnosis of PEM fuel cell stack
}

Zhixue Zheng, Simon Morando, Marie-Cécile Péra, Daniel Hissel, Laurent Larger, Romain Martinenghi, Antonio Fuentes

\section{- To cite this version:}

Zhixue Zheng, Simon Morando, Marie-Cécile Péra, Daniel Hissel, Laurent Larger, et al.. Brain-inspired computational paradigm dedicated to fault diagnosis of PEM fuel cell stack. International Journal of Hydrogen Energy, 2017, 42 (8), pp.5410-5425. 10.1016/j.ijhydene.2016.11.043 . hal-02131009

\author{
HAL Id: hal-02131009 \\ https://hal.science/hal-02131009
}

Submitted on 6 Oct 2021

HAL is a multi-disciplinary open access archive for the deposit and dissemination of scientific research documents, whether they are published or not. The documents may come from teaching and research institutions in France or abroad, or from public or private research centers.
L'archive ouverte pluridisciplinaire HAL, est destinée au dépôt et à la diffusion de documents scientifiques de niveau recherche, publiés ou non, émanant des établissements d'enseignement et de recherche français ou étrangers, des laboratoires publics ou privés.

\section{(c)(1)}

Distributed under a Creative Commons Attribution| 4.0 International License 


\title{
Brain-inspired computational paradigm dedicated to fault diagnosis of PEM fuel cell stack
}

\author{
Zhixue Zheng $^{\mathrm{a}, \mathrm{b}}$, Simon Morando ${ }^{\mathrm{a}, \mathrm{b},{ }^{*}, \text { Marie-Cécile Pera }}{ }^{\mathrm{a}, \mathrm{b}}$, Daniel Hissel ${ }^{\mathrm{a}, \mathrm{b}}$, \\ Laurent Larger $^{\mathrm{b}}$, Romain Martinenghi ${ }^{\mathrm{b}}$, Antonio Baylon Fuentes ${ }^{\mathrm{b}}$
}

a FCLAB Research Federation, FR CNRS 3539, University of Franche-Comte, 90010 Belfort Cedex, France

${ }^{\mathrm{b}}$ FEMTO-ST, UMR CNRS 6174, University of Bourgogne Franche-Comte, 25030 Besançon Cedex, France

\begin{abstract}
Features such as low greenhouse-gas emission, high energy efficiency and operating sta-bility make fuel cell (FC) an attractive power source for a wide variety of applications. Nevertheless, to achieve its commercialization, durability and reliability remain big challenges. This work aims at developing an efficient data-driven fault detection and identifi-cation methodology through the use of a recently proposed brain-inspired computational paradigm, Reservoir Computing (RC). The considered "Reservoir" is made of a particular class of complex dynamics emulating a virtual neural network, and modeled by a nonlinear delay equation. This original and experimentally compatible approach indeed demonstrated recently excellent performances on complex nonlinear problems such as classification and prediction tasks. In this work, a first attempt is made to introduce the RC method into the field of FC diagnosis. Targeted fault types include CO poisoning, low air flow rate, defective cooling and natural degradation. Experimental results show the simplicity and efficiency of RC method to discriminate the abovementioned health states. Moreover, the influence of four key RC parameters and also of the learning database is investigated in order to explore the possibility of further facilitating and generalizing the RC approach.
\end{abstract}

\section{Introduction}

Fuel cell (FC) is considered as one of the most promising power generation systems in the near future [1-3]. As a clean and efficient electro-chemical power converter, FC converts directly the chemical energy of the fuel (e.g. hydrogen) into electricity without internal combustion. Their only byproducts are water and heat in the case of pure hydrogen being supplied. Compared with conventional power plants, the principle advantages of FC include:

- High energy efficiency, twice as efficient as the conventional internal combustion engine [4];

- Zero or near zero greenhouse-gas emission according to the FC types and the production way of hydrogen;

- Operating stability and maintenance simplicity sincere few moving parts exist in the system, thus providing less vibrations and highly reliable operation [5];

\footnotetext{
* Corresponding author. FEMTO-ST, UMR CNRS 6174, University of Bourgogne Franche-Comte, 25030 Besançon Cedex, France.

E-mail address: simon.morando@femto-st.fr (S. Morando).
} 
- Generating electricity as long as the fuel is supplied, unlike the batteries [5];

- Coping with the intermittency of the renewables such as wind and solar power, without geographic limitations [6].

Among the different FC technologies, polymer electrolyte membrane (or proton exchange membrane) fuel cells (PEMFCs) have drawn the most attention from both the academic and industrial researchers [1-3,7]. The dominance of PEMFC technology can be reflected in terms of the number of FC system shipments in recent years, which accounts for $88 \%$ of the total FC shipments [8]. Generally, they operate at relatively low temperature $\left(<120^{\circ} \mathrm{C}\right)$ which allows them to start up quickly. Moreover, high compactness and low weight make them perfect for transportation and portable applications [9]. Despite of their desirable properties, one of the primary barriers nowadays for their commercialization is the reliability and durability [1]. A lifetime of $2,500 \mathrm{~h}$ for transportation PEMFC stack was reported in Ref. [10], which reaches only the half of the DoE 2010 targets (5,000 h for transportation) [11]. Fault diagnosis is therefore of high necessity in reinforcing the reliability and improving the durability of PEMFCs.

In this paper, we present a framework based on RC for fault detection and isolation of PEMFC stacks. Firstly, a brief presentation of PEMFC fault diagnosis context is realized in the 2nde section. Then, an introduction to the RC method is made in "Introduction to reservoir computing (RC)" Section in order to give a clear idea of this method. "PEMFC system description" Section deals with how to utilize RC for fuel cell diagnosis and its main interests. "Reservoir computing (RC) for fuel cell diagnosis" Section presents the targeted PEMFC system and four involved fault types. Diagnosis results are illustrated and further analysis is made in "Reservoir computing (RC) for fuel cell diagnosis" Section. The final section ends with a conclusion and perspectives.

\section{Context of the study}

\section{PEMFC diagnosis}

Many works on the PEMFC diagnosis are presented in Refs. $[12,13]$. Methods based on the parameters' identification are often used. The EIS based models extracts descriptors from EIS to monitor and make a diagnosis online. This method can be used for the detection of water management issues, and for the analysis of aging and degradation [14-16].

In Ref. [14], a robust control is applied to the fuel cell. For this, the controller needs to know precisely the operation conditions of the PEMFC, obtained by online spectroscopy. In Ref. [15], PEMFC monitoring of flooding and drying is performed using a model-based approach coupled with EIS. A study of the impedance response of the fuel cell as a function of the relative humidity in the inlet gas was performed. The parameters of an electric equivalent circuit were then extracted from the obtained data. In Ref. [16], one PEMFC cell at high temperature was analyzed in different operating conditions using impedance spectroscopy, with the main aim of providing a data set for future fuel cell diagnostic systems. The proposed model is able to provide a good estimation of the experimental data in all operating modes. It was also shown that the temperature has an influence on the ohmic resistance and, on the other hand, on the charge transfer resistance. The gas transfer resistance showed a strong dependence on current and a weaker dependence to the temperature.

In Ref. [17], a diagnostic method is performed using a model based on the relative sensitivity of the fault. The advantage of this method is that it does not require knowledge about the fault severity to provide a diagnosis. To test this methodology, a PEMFC simulator has been developed. This simulator has been modified to include a different set of fault condition. All simulated faults have been tested with this diagnostic method, which has managed to correctly detect the simulated faults. The simulated faults taken into account were: failure on the compressor motor, flooding fault in the cathode compartment, air leakage to the cathode and problem in the temperature control.

The work depicted in Ref. [18] is focused on calculating residuals using an observer. The isolation of defects is achieved by using structured residues once the fault has been detected. The proposed algorithm is able to identify and evaluate multiple defects sensors presented at different times. The algorithm is tested on a commercial fuel cell where a set of fault scenarios is considered.

In Ref. [19], the CVA (for Canonical Variable Analysis) with a relatively small order is used. The CVA is similar to PCA (Principal Component Analysis), a geometric and statistics method to analyze data. Two concepts are then tested, a Kalman filter and a reverse model, which show how models of the CVA state space can be used for the diagnosis of nonmeasurable inputs.

These models are used to represent the non-linear behavior of a PEM in operating range similar to load cycles of vehicle driving cycles.

The PCA to estimate a 50 cell PEMFC health status offline is realized in Ref. [20]. This analysis is based on data provided from temperature, voltage, current, flow and humidity sensors. In this work, the PCA makes it possible to exploit the correlations between variables and the similarities between the measurements at time t. To complete this process, an empirical model of PEMFC is proposed connecting the various input parameters to the cell voltage using a multiple linear regression.

Fuzzy logic was used in Ref. [21]. A diagnostic methodology based on fuzzy classification (FCM for Fuzzy c-Means) is developed in combination with data from the EIS. This method allows to develop a diagnostic methodology online with the ability to distinguish different degrees of flooding and drying inside the PEMFC.

Bayesian methods to detect a drying up and flooding the PEMFC offline using data from the EIS are proposed in Ref. [22]. A naive Bayesian classifier was chosen as the approach. Twelve input variables allow differentiation between six operating modes: minor drying, moderate drying, light flooding, minor flooding, moderate flooding, and a normal mode.

After a study of the parameters that can influence the correct classification rate, optimized classifier is proposed, able to detect $91 \%$ of the samples of the validation database.

Methods from signal processing are also used for the diagnosis of PEMFC with firstly the use of fast Fourier 
transform (FFT). In Ref. [23], FFT analysis on the voltage and pressure information to detect a flooding phenomenon on a stack of 10 cells is realized. The dominant frequency drop of the pressure signal is used as an indicator of the behavior of water in the cathode and in the anode, thereby predicting the voltage variation of the fuel cell.

The wavelet transform is used in Ref. [24], in order to diagnose the health of a fuel cell only with the analysis of its voltage signal which is the easiest and least expensive variable to monitor in a PEMFC system. The feasibility and reliability of this method make it possible to correctly classify the different following states of the PEMFC: drying and flooding cells.

Finally, in Ref. [25], the authors demonstrated the possibility of using an ANN (Artificial Neural Network) based procedure for diagnosing water management issues, including drying out and flooding. Based on the recurrent ANN (RNN) based model, the threshold functions were defined and applied on the residuals between the actual and the model outputs (i.e. stack voltage and pressure drop) to discriminate both faults. Hamming neural networks are used in Ref. [26] to estimate offline the health of a 20 cell PEMFC, each cell being monitored, with the voltage sensors and current information only. A diagnostic approach based on Hamming neural network for the identification of appropriate parameters of fuel cell model is proposed to diagnose the state of health (SOH) of a PEMFC. The output voltages of a 20 PEM fuel cell were measured, in addition to the parameters of the model, in order to be used as the training and validation dataset. This subsection allows to highlight the use of artificial neural network in the diagnosis of PEMFC. However, the next section presents other applications of ANN in the field of the study of PEMFC, to insist on the popularity and effectiveness of ANN in the different areas of FC studies.

\section{Use of neural networks in various PEMFC research domain}

As mentioned in the previous section, among the various methods proposed in the literature, ANN are present in many fields of study related to the PEMFC, as an efficient method to grasp its complexity. The focus are particularly placed on the modeling and the control of PEMFC fields.

Concerning the modeling of PEMFC field, in Ref. [27], the authors propose a neural network modelling approach for the mechanical nonlinear behavior of a PEMFC. An experimental set is designed for this purpose: a fuel cell system in operation is subjected to random and swept-sine excitations on a vibrating platform in three axes directions. The mechanical response is measured with accelerometers. A neural network containing 18 hidden neurons is then build with the collected data-based. The results obtained are very interesting, and make it possible to optimize the position of available sensors in order to get the most sensitive mechanical response to loads. A different model is proposed in Ref. [28], where three different kind of neural networks (Multilayer Perceptron (MLP), Generalized Feedforward Network and Jordan and Elman Network) are designed in order to model a PEMFC stack performance according to the temperature. The influence of temperature of two periods was studied: the temperature in the conditioning period and temperature when the fuel cell was operating. A comparison between the three types of neural model tested is realized and the authors conclude than the MLP gives better results for this application. Jemei et al. 2008 [29] proposed a static and dynamic model of a PEMFC system by using ANN, particularly feedforward type. Stack current, stack temperature, air humidity, hydrogen and oxygen flow are inputs and the stack voltage is the output. A good consistency between the proposed ANN model and the experimental result were observed, i.e. the error rate is less than $1 \%$ for a single cell compared with $5 \%-10 \%$ given by mathematical or hybrid models.

Obviously, the ANN are also used for the control of a PEMFC [30]. In this article, a recurrent neural network is used to control the water management in PEMFC. The water concentration in cathode is controlled and the humidity fluctuation are reduced at the cathode. Simulation results showed that a controller based on RNN has shorter response time than PID controller. These results are obtained only with simulation in Matlab-Simulink environment. In Ref. [31], a neural networks (NN)-based active and reactive power controller of a standalone PEMFC power plant (FCPP) is proposed. The proposed controller is used to modify the inverter modulation index and the phase angle of the ac output voltage to control the active and reactive power output from the FCPP to match the terminal load. The proposed controller and the fuel cell power plant model have been tested with simulations and the data used corresponds to an active and reactive load demands of a single-family residence.

Numerous studies have proved the ANN strong capability in the modelling or pattern recognition of nonlinear complex systems [28-32]. It can indeed approach any nonlinear mapping with arbitrary accuracy when properly trained [33]. Compared with the physical models, the ANN method provides an attractive alternative since it doesn't need huge knowledge about the underlying physical processes. Moreover, an accurate identification of inner physical parameters is not required.

Despite its widely acknowledged potential and a number of successful applications reported in the literature, ANN's impact in PEMFC domain still remains limited. The main reason lies in the fact that ANN, especially the RNN type is inherently difficult to train. The generally adopted gradientdescent based methods aim at iteratively reducing the training error, which always suffer from the following shortcomings:

(1) The low convergence rate and the high computational training costs. The update of a single parameter is usually computationally expensive. It may require many update cycles. Consequently, ANN training is feasible only for relatively small neural networks (in the orders of tens of units) [33].

(2) The existence of bifurcations [34]. In the course of learning, the network dynamics may experience bifurcations due to the discontinuities at some points (called "bifurcation points") in the parameter space. This may result in undesirable consequences like the explosion of the error gradient, the jump of the network rate. As a consequence, the convergence of the training progress cannot be guaranteed, or even converged, local optima may be obtained. 
These may hinder RNN's application especially in real-time scenarios. A recently proposed, brain-inspired computational paradigm called Reservoir Computing (RC) is reported to have overcome the shortbacks of traditional ANNs like high computational training cost and low convergence rate. Excellent performances of RC have been achieved in the literature, moreover, with hardware implementation instead of the usual software only solution, on complex non-linear problems such as modeling, classification and prediction tasks [32,35]. Meanwhile, in the field of fuel cell fault diagnosis, RC is still a relatively new concept. In regard of this, it is very interesting to make such an attempt to adapt the RC paradigm for data-driven fault diagnosis of PEMFCs.

\section{Introduction to reservoir computing (RC)}

\section{Birth of RC}

To have a clear understanding of reservoir computing, recurrent neural network as its originated theoretical ground is unavoidable to be introduced. Generally, according to the network topology, ANN can be divided into two types: feedforward and recurrent network, which is illustrated in Fig. 1 (a) and (b).

Feedforward type (FNN), often referred as multi-layer perceptron (MLP), is the most commonly used topology in the literature. It is essentially a non-linear functional mapping between a set of input and output variables. Due to its feedforward structure and non-dynamic nature, its applications have been mainly restricted to the processing of non-temporal problems [36]. Meanwhile, in RNN structure, the presence of recurrent connections makes the information not only flow forwardly, but also circulated inside the network. Thus, the current information is integrated with that of the previous steps. Consequently, the network is a nonlinear mapping of both the current input and the input history. By virtue of this property, RNN is able to solve inherently temporal problems, e.g. speech recognition and temporal prediction. Theoretically, RNN can be regarded as a universal approximator of dynamical systems under mild and general assumptions. That can also explain perfectly why RNN gains a higher popularity in the practical applications especially when dealing with dynamic problems.

Nonetheless, as explained in the introduction, the traditional RNN training methods may suffer from the low convergence rate, high computational cost and local optima. From the year 2000 on, a new trend for understating, designing and training RNN has been formed to overcome its limitations. Two typical methods are Echo State Network (ESN) and Liquid State Machine (LSM), which were proposed independently by Prof. Jaeger in 2001 [37] and Prof. Maas et Prof. Markram in 2002 [38]. The two methods, combined with the later appearing Back-Propagation De-Correlation (BPDC) learning rule were unified into a common research stream [39]. That is Reservoir Computing (RC).

\section{Fundamental idea of $R C$}

The fundamental idea can be vividly revealed by the concept "reservoir". A reservoir is regarded as a container of abundant dynamic transient states. Indeed, a reservoir usually contains $10^{2}$ to $10^{3}$ internal neurons, which is much more than the traditional RNN (typically tens of neurons). If the reservoir satisfies certain properties, a linear combination of the transient states could be sufficient to solve complex nonlinear problem with an excellent performance [37].

The main differences of RC paradigm compared with the traditional RNN training methods consist in: firstly, the internal network structure is assumed to be fixed and isn't optimized according to the training process. Thus, the network dynamics are not affected during training. Secondly, the training process is strongly simplified. It is reduced to the determination of output weights, which can be easily calculated by linear regression techniques [33]. A schematic illustration is shown in Fig. 2. In this figure, the arrows represents the optimization of the weight values realized according to the error between the output and the target signal. As we can see, just one optimization is needed for the RC paradigm.

An interesting view can be presented when RC is compared with the kernel methods, e.g. support vector machine. The key idea behind kernel methods is mapping the
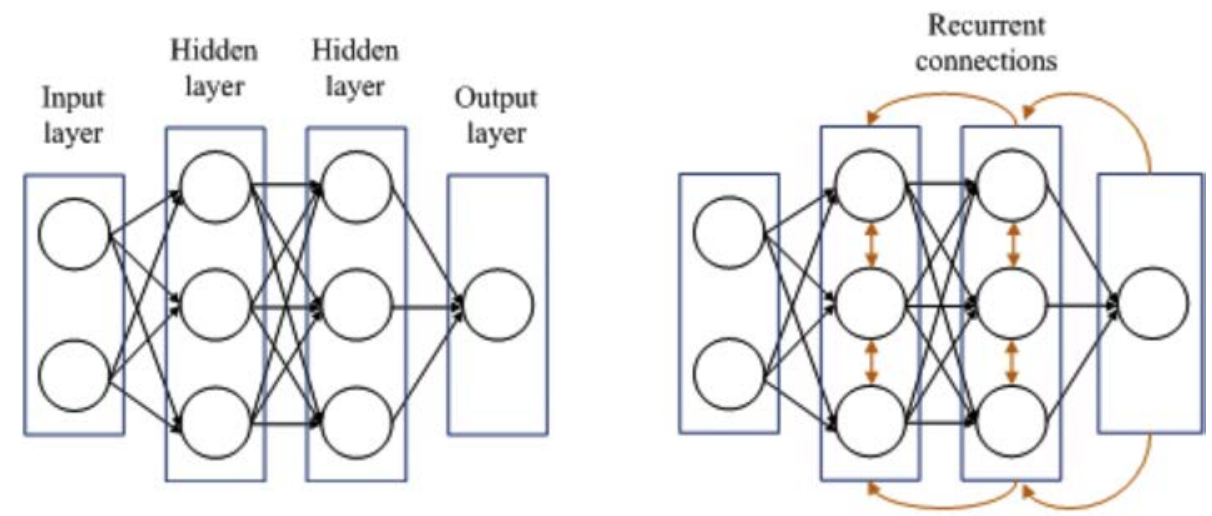

(a) Feedforward neural network (b) Recurrent neural network

Fig. 1 - Different network topologies. 
A.

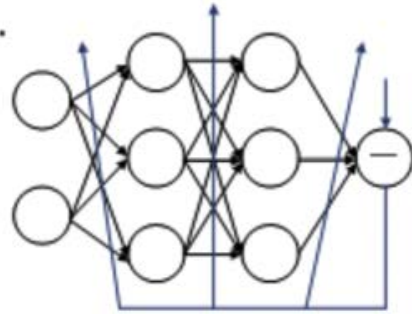

B.

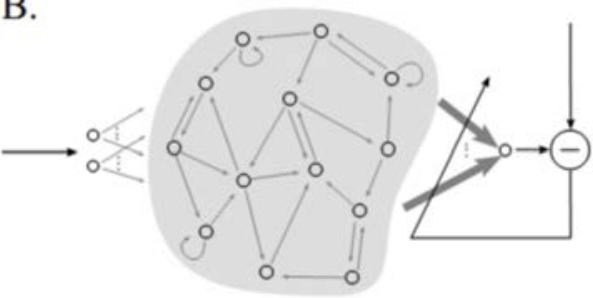

Fig. 2 - Traditional RNN and RC paradigm. (A)Traditional gradient-descent based RNN training methods adapt all connection weights, including input-to-RNN, RNN-internal, and RNN-to-output weights. (B) In RC, only the RNN-to-output weights (bold arrows) are adapted [34].

low dimensional input space into a high dimensional feature space by some kernel functions, where the classification or regression is greatly facilitated. The functionality of the reservoir here is similar to that of kernel function. In RC, the input signal is non-linearly mapped into a high dimensional reservoir state through the transient response of the reservoir [33], where the RC output is a linear combination of the transient states with adjustable weights. A graphic illustration is shown in Fig. 3.

Due to its simplicity of use and excellent performances, RC has quickly gained a high popularity in various practical applications, especially in the field of machine learning. For instance, the word error rate was brought to $0 \%$ from the previous $0.6 \%$ concerning the speech recognition task [39]. A $0 \%$ test error has been achieved on the Japanese Vowel benchmark while the previous best test error is $1.8 \%$ [40]. The non-linear wireless channel equalization has been improved by two orders of magnitude, meanwhile the prediction of chaotic dynamic utilizing RC has been improved by three orders of magnitude compared with previous methods [41]. Furthermore, in a recent international financial forecasting competition it wins the contest. ${ }^{1}$

\section{RC topologies}

Generally, RC consists of three distinct parts: an input layer, a reservoir and an output layer. The input layer is connected with the reservoir via a randomly generated input weight matrix $\left(\mathrm{W}^{\mathrm{I}}\right)$, which remains unchanged during training. The reservoir contains a large number of randomly interconnected nodes (via $W^{\mathrm{D}}$ ) which are also left untrained [33]. When excited by the input signals, the reservoir exhibits complex transient dynamics, which are further read out by the output layer via an output weight matrix $\left(\mathrm{W}^{\mathrm{R}}\right)$.)

A discrete-time RC with a basic architecture consists of $K$ inputs, $N$ internal nodes and $L$ outputs is represented in Fig. 4 (a). For this architecture, the reservoir is set randomly, in contrast to Fig. 4 (b), where the reservoir structure is ordered. At time step $n$, the inputs are $u(n)=\left[u_{1}(n), \ldots u_{K}(n)\right]$, internal nodes are $x(n)=\left[x_{1}(n), \ldots x_{N}(n)\right]$, and the outputs are $y(n)=\left[y_{1}(n), \ldots y_{L}(n)\right]$.

At time step $n$, the activation state of the internal nodes is a non-linear transformation of both the current inputs and the previous states of the nodes and outputs.

\footnotetext{
${ }^{1}$ http://www.neural-Forecasting-competition.com/NN3/index. htm.
}

$x(n)=f_{N L}\left[W^{D} \cdot x(n-1)+W^{I} \cdot u(n)+W^{F B} \cdot y(n-1)\right]$

where $f_{\mathrm{NL}}(\cdot)$ is the neuron activation function which is usually non-linear, $\mathrm{W}^{\mathrm{I}}$ is the input weight matrix, $\mathrm{W}^{\mathrm{D}}$, is the reservoir weight matrix of the interconnected nodes, $\mathrm{W}^{\mathrm{FB}}$ is an optional output feedback weight matrix. The output is further calculated by a simple linear combination of $x(n)$ :

$y(n)=W^{R} \cdot x(n)=\sum_{i=1}^{N} w_{k i}^{R} \cdot x_{i}(n)$

with $\mathrm{W}^{\mathrm{R}}$ the trained output weight matrix.

Classical RC is largely driven by a series of randomized model building stages, which could be unstable and hard to understand, especially for fault diagnosis. In this paper, we propose to use a specific architecture of RC, which is comprised of a single nonlinear node with delayed feedback, as illustrated in Fig. 4 (b). This novel architecture was proposed by Appeltant et al., in 2011 [35], and its experimental implementation of RC was also realized firstly. Afterwards, several works have been performed based on this specific reservoir architecture and considerable successes were reported on a variety of practical tasks [30,34,45]. Compared with the classical randomly created RC, it has a specific reservoir design and a much simplified structure, which greatly facilitates the hardware implementation. Furthermore, it favors a deeper understanding of the interplay of dynamical properties and reservoir performance [35]. Meanwhile, this architecture is reported to have as good performance as traditional RCs for certain tasks [32].

The reservoir is an assembly of virtual nodes, which are obtained by dividing the delay loop into $\mathrm{N}$ equally distanced intervals. The time interval between each two adjacent nodes is $\delta_{\tau}$ (also called node distance). Thus, the total delay time of the delay loop is $\tau_{\mathrm{D}}=N \times \delta_{\tau}$. The nodes composing the reservoir here are in fact the delay dynamics at different temporal positions. They have the same functionality as the traditional nodes. That's also why they are named "virtual node". In order to achieve the optimal performance of RC, the choice of node distance $\delta_{\tau}$ is crucial. Usually, $\delta_{\tau}<\mathrm{T}_{\mathrm{R}}$, with $\mathrm{T}_{\mathrm{R}}$ being the characteristic time of the non-linear node. In this regime, the states of the virtual nodes depend on the states of the neighboring nodes instead of behaving like $N$ independent nodes. Interconnected in this way, the entity of virtual nodes serves as a reservoir [35]. In "PEMFC system description" Section, more details of utilizing this novel architecture for fault diagnosis are presented. 

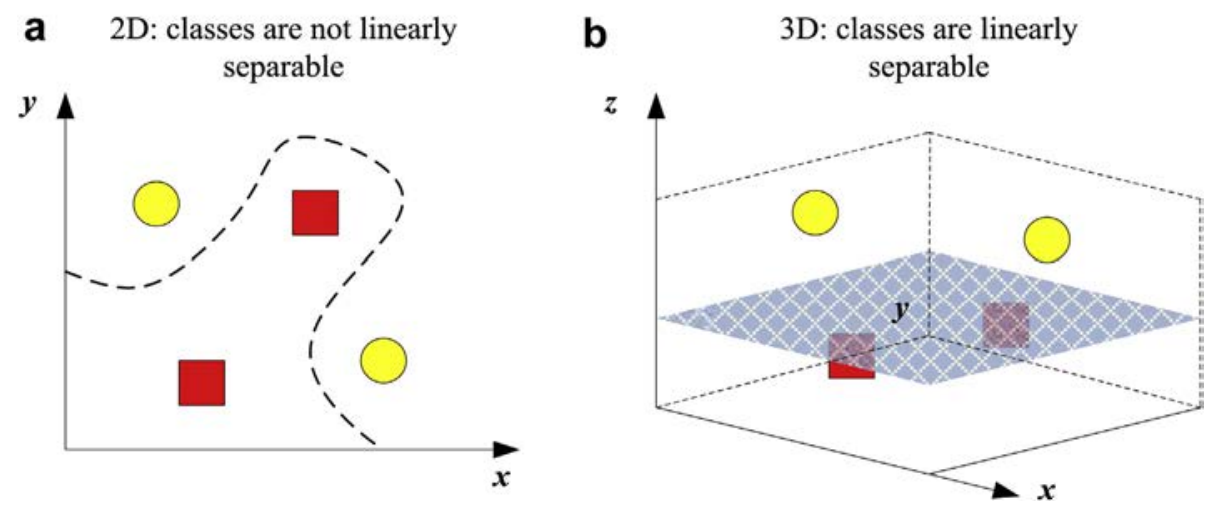

Fig. 3 - Illustration of linear reparability, adapted from Ref. [24]. (a) The two classes are not linearly separable in a two dimensional space. (b) By a non-linear mapping into a three dimensional space, the two classes are separable by a linear hyperplane.

\section{PEMFC system description}

\section{Description of the test bench}

The experimental tests were performed on a $1 \mathrm{~kW}$ fuel cell test bench developed in the laboratory. A detailed description of the test bench can be found in Ref. [45]. The purpose of the tests was to introduce different controlled health states into a PEMFC stack by configuring various operating parameters, e.g. gas flows, stack temperature, humidity levels. A simplified schema of the test bench is illustrated in Fig. 5. The gas circuits include the hydrogen and the air circuit. The gas flow rate and pressure can be controlled separately thanks to a flow rate controller and a back pressure valve. Before entering the stack, both the hydrogen and the air can be humidified or kept dry. A cooling water circuit which consists of a cold source and a hot source was applied to manage the stack temperature. The stack outlet water temperature is classically considered as the representative the actual stack temperature.
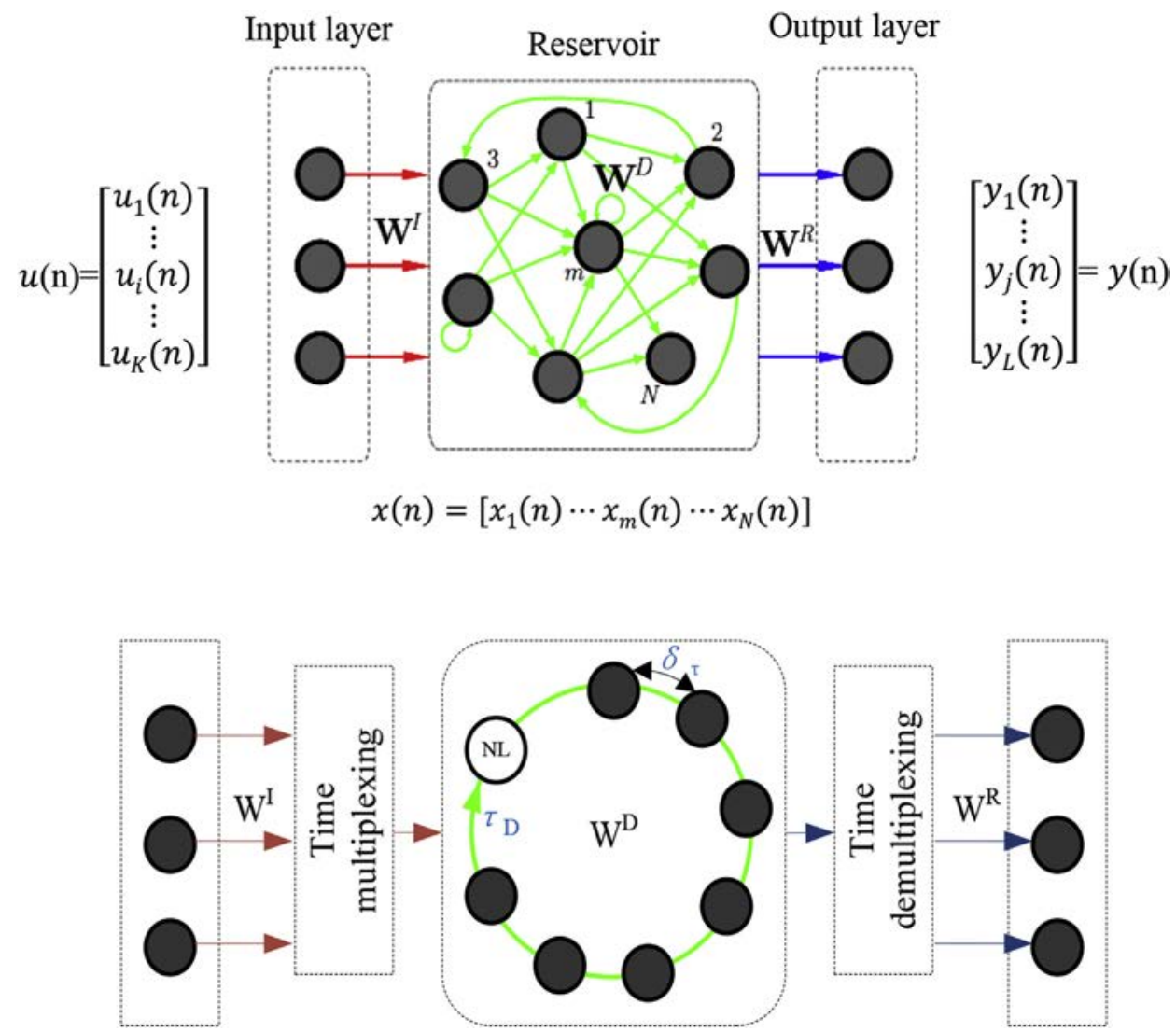

Fig. 4 - Sketch of RC schemes. Classical RC scheme (top), Scheme of RC utilizing a nonlinear node with delayed feedback (bottom). 
In this work, a 8-cell PEMFC stack was investigated, supplied by CEA, with its basic information listed in Table 1 . The stack works nominally at a current of $110 \mathrm{~A}$, as its active surface is equal to $220 \mathrm{~cm}^{2}$.

\section{Four degrading health states}

The set of tests carried out and focused on in this paper includes four degrading operating conditions:

$\checkmark$ Low cathode stoichiometric ratio (FSC). FSC plays an import role in the health management of fuel cell systems. It influences the oxygen availability as well as the humidity of the membrane. Compared with high FSCs, low ones could lead to more rapid and severe degradation of the fuel cell, as it is an incentive factor of cathode water flooding and cathode starvation. As a result, evident fluctuations could be normally observed in the stack voltage signal when the stack current is kept constant. In this study, different values of FSC were applied on the stack in order to study its influence on the stack performance. Stable stack voltage under the lowest FSC which equals 1.2 was recorded for final comparison.

$\checkmark$ Defective cooling system. The effect of cooling system is studied here considering its connection to the stack temperature. Defective cooling could result in excessive stack temperature and may further introduce the membrane drying phenomenon. In this study, the flow rate of the water circulating in the cooling system (FRW) was reduced gradually in order to observe its impact on the stack performance. Stable stack voltage under the lowest water flow rate of $0.5 \mathrm{Nl} / \mathrm{min}$ (Normal liters per minute) was acquired for further evaluation.

$\checkmark$ CO poisoning. CO poisoning is considered as one of the most common faults for PEMFCs which use platinum as their catalyst. $\mathrm{CO}$ in the hydrogen flow is preferentially absorbed to the platinum and thus block or limit the active sites. Normally, the poisoning effect is slow and reversible if the anode is exposed to dioxygen. During the fault
Table 1 - Description of the fuel cell and its nominal operating conditions.

\begin{tabular}{ll} 
Parameters & Value \\
\hline Number of cells & 8 \\
Electrode active surface & $220 \mathrm{~cm}^{2}$ \\
Coolant flow: water & $2 \mathrm{Nl} / \mathrm{min}$ \\
Anode stoichiometry & 1.5 \\
Cathode stoichiometry & 2 \\
Absolute pressure of input $\mathrm{H}_{2}$ & $150 \mathrm{kPa}$ \\
Absolute pressure of input air & $150 \mathrm{kPa}$ \\
Maximum pressure difference & $30 \mathrm{kPa}$ \\
Temperature of output cooling circuit & $80{ }^{\circ} \mathrm{C}$ \\
Anode relative humidity & $50 \%$ \\
Cathode relative humidity & $50 \%$ \\
Current (Istk) & $110 \mathrm{~A}$ \\
Current density & $0.5 \mathrm{~A} / \mathrm{cm}^{2}$ \\
\hline
\end{tabular}

process, a slow decline of the stack voltage can be observed. In our test, a low concentration of CO (10 ppm) was mixed with pure hydrogen to supply the anode side of the stack. The test was carried out by operating the stack in the presence of $\mathrm{CO}$ during $2 \mathrm{~h}$ until the stack reached a steady state.

$\checkmark$ Continuous aging life test. Compared with the abovementioned faults, the natural aging effect causes the slowest performance degradation. A $124 \mathrm{~h}$ continuous test under nominal conditions was conducted on the stack to observe the natural degrading effect under the time evolution. A degradation rate of $1.3 \mathrm{mV} / \mathrm{h}$ is observed on the stack voltage during the whole process. Specially, the stack voltage at the end of the aging test is collected for comparison.

A set of stack voltages recorded under normal and the four degrading conditions are plotted in Fig. 6. The acquisition frequency is $11 \mathrm{~Hz}$. Each sequence consists of 2000 points and lasts for about $3 \mathrm{~min}$. Different behaviors of stack voltage under the time evolution can be visually observed.

At first sight, CO poisoning after 2 h' test seems to cause the most severe performance degradation of the fuel cell, as it

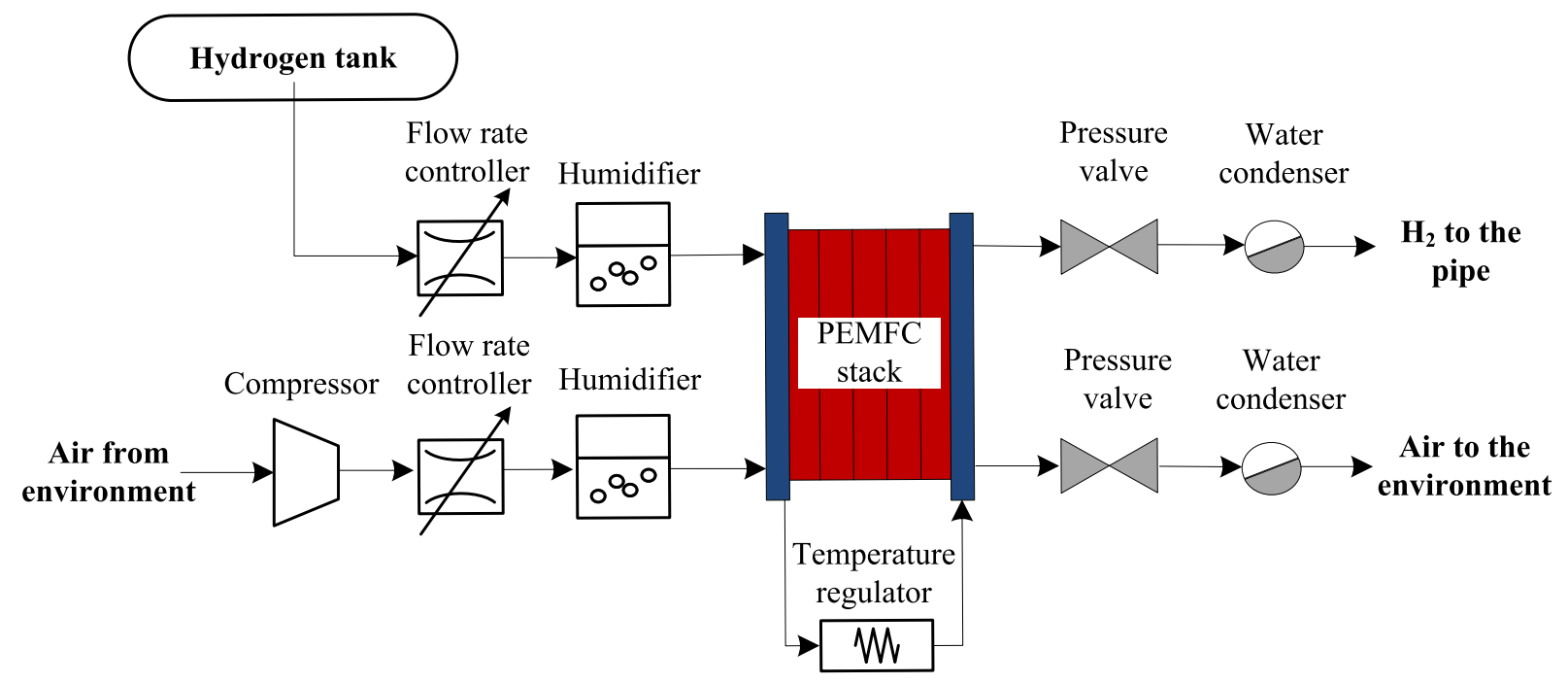

Fig. 5 - Simplified diagram of the PEMFC system. 
(a) Normal view of stack voltage

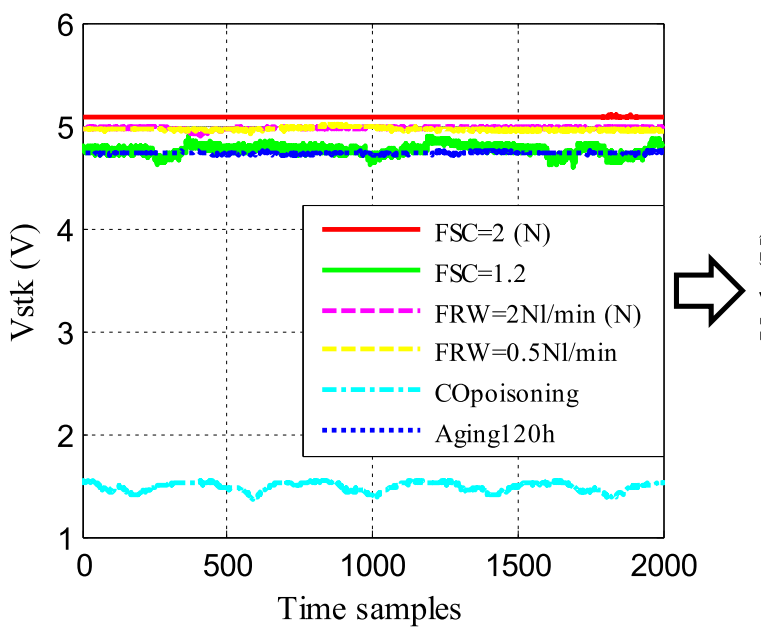

(b) Enlarged figure

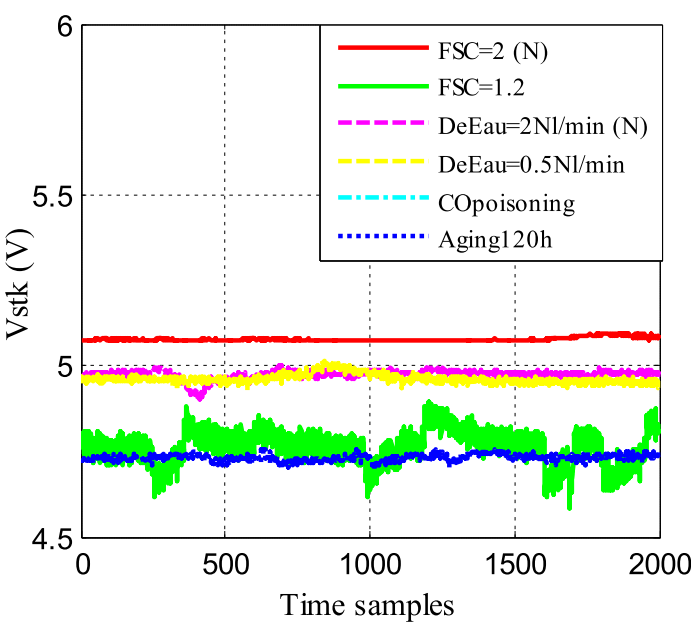

Fig. 6 - Stack voltages under normal and four degrading conditions.

resulted in the lowest stack voltage which is about $1.5 \mathrm{~V}$, whereas the other conditions correspond to a value around $5 \mathrm{~V}$. In order to show more details about the stack voltage under the other operating conditions, a further enlarged figure was illustrated in Fig. 6 (b). It can be found that low FSC and aging generate similar average stack voltage, while their shapes differ significantly. The flow rate of cooling water seems to have little influence on the stack voltage as the two voltages under $\mathrm{FRW}=2 \mathrm{Nl} / \mathrm{min}$ and $1 \mathrm{Nl} / \mathrm{min}$ have similar magnitude and temporal variations.

\section{Reservoir computing (RC) for fuel cell diagnosis}

\section{Fault diagnosis principle}

The objective of this section is to utilize RC directly as a fault detector and classifier to discriminate different health states of the PEMFC stack, without the necessity of modelling the whole system. In the first part of this work, stack voltage is applied as the only input signal of the RC for fault diagnosis. The stack voltage signal is considered as an informative signal which can reflect the FC health state. In Ref. [48], the voltage signals were applied as database to discriminate influence of air flow rate, hydrogen flow rate and gas pressure. In Ref. [29], the stack voltage drop is considered as a common consequence of both drying out and flooding. In Refs. [47-49], fault classification was successfully performed in a cell-voltage generated space.

The principle idea of the diagnosis method, illustrated in Fig. 7, is based on the analysis of the frequency response of the stack under fault. As a fault occurs, the physical state of the FC changes, and therefore the frequency content of its voltage response changes as well. The diagnosis method relies then on four basic steps:

(1) Signal preprocessing. The voltage signal is firstly transformed into a time-frequency representation by Short Time Fourier Transform (STFT). Simply speaking, through a moving window process, the original voltage signal in time domain is broken up into a set of small segments. Each segment is then processed by traditional Fast Fourier Transform (FFT). It provides the information about the evolution of each frequency component and its instantaneous strength (in form of power spectral density) along the time axis.

(2) RC input. In this step, an input weight matrix $\mathrm{W}^{\mathrm{I}}$ is created randomly with a low sparsity ( 0.1 with non-zero elements filled with \pm 1 ). It performs a random selection of the frequency components along the time samples. The final input which activates the internal nodes is the input signal matrix $M_{u}$, which is a multiplication of the input weight matrix $\mathrm{W}^{\mathrm{I}}$ and the time-frequency representation $M_{c}: M_{u}=W^{I} \times M_{c}$.

(3) RC nonlinear transformation. It is performed by a dynamic function which is physically based on an optoelectronic implementation, with more details described in Ref. [32]. The transformation plays the role of injecting the preprocessed signal into the nonlinear function and generating the corresponding reservoir states which are further read out in the output layer.

The utilized non-linear dynamic function $f_{\mathrm{NL}}$ is expressed as [32]:

$\mathrm{T}_{R} \frac{d x(t)}{d t}+x(t)=\beta \sin ^{2}\left(\mu x\left(t-\tau_{D}\right)+\rho u\left(t-\tau_{D}\right)+\varnothing_{0}\right)$

where $T_{R}$ is the internal characteristic time scale of the nonlinear dynamics. It determines the coupling extent of the extent of the internal virtual nodes (or the reservoir connectivity) through its impulse response. The longer $T_{R}$ relatively to the node distance $\delta_{\tau}$ is, the more connected consecutive nodes are [32]. $\beta$ is the nonlinear gain. Normally its value should be smaller than 1 in order to avoid destabilization of the dynamics. $\phi_{0}$ is the offset phase, which determines the mean operating point along the nonlinear function. For instance, $\phi_{0}$ close to 0 or $\pm \pi / 2$ gives average operating point along the parabola, while $\phi_{0}$ around $\pm \pi / 4$ gives a linear average operating point. $\mu$ is the 


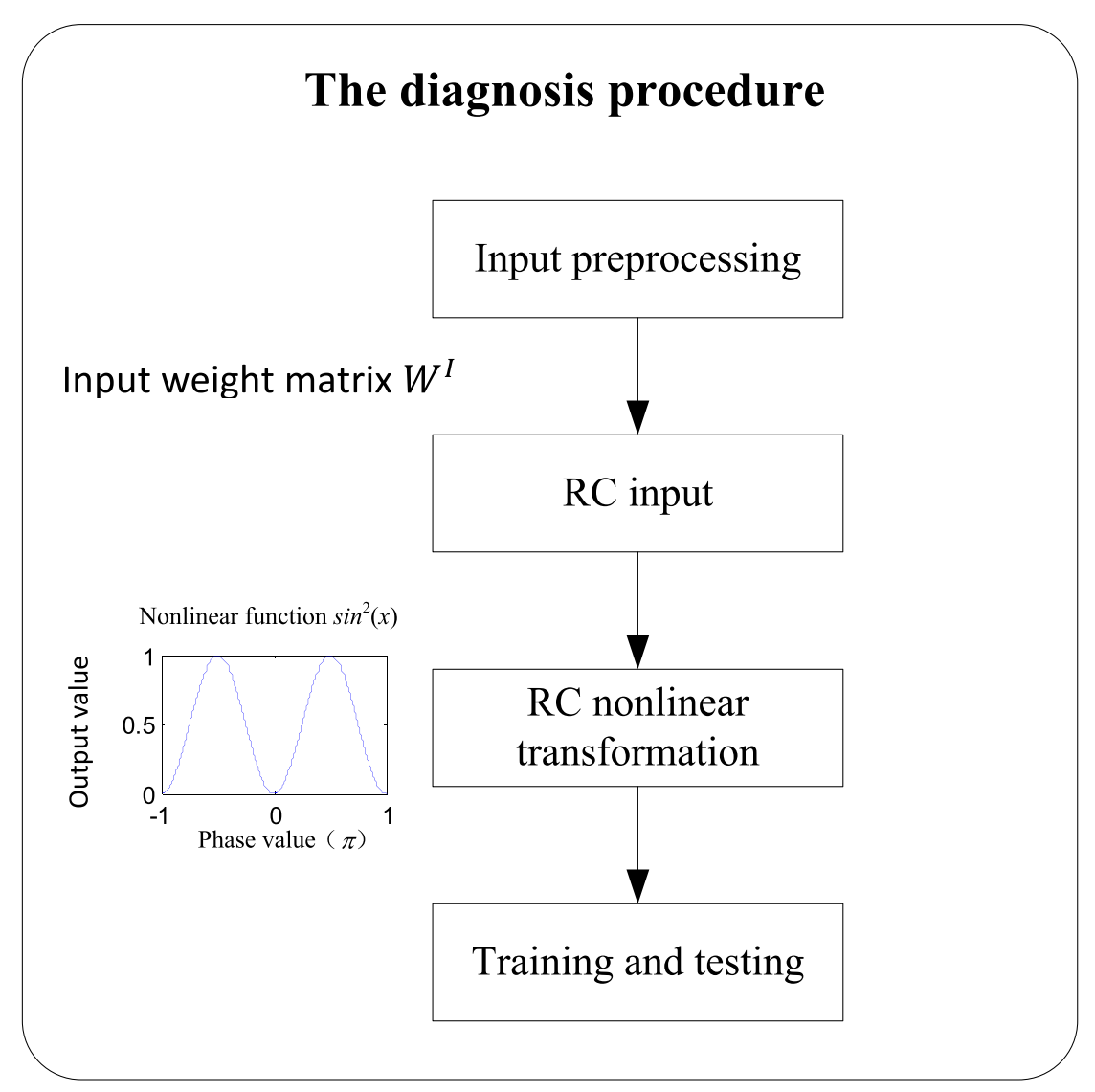

Fig. 7 - Principle of the RC diagnosis method.

feedback scaling. It is a binary parameter (i.e. 0 or 1 ) which controls the open or closed operation of the feedback loop. Normally, a feedback operation is adopted, i.e. $\mu=1$. $\rho$ is the input scaling factor which corresponds to the amplifier of the input signal. It represents the relative weight of the input information versus the feedback signal $x\left(t-\tau_{\mathrm{D}}\right)$.

To summarize, when utilizing RC for a specific task, a number of key parameters need to be adjusted in order to obtain good performance. These include the non-linearity gain $\beta$ and the offset phase $\phi_{0}$ which are related to the non-linear dynamics, input scaling $\rho$ and the regression parameter $\lambda$, given that the fundamental structure of the reservoir is set (inc. $T_{R}, \tau_{D}$ and $N_{n}$ ).

(4) Training and testing. The objective of the training procedure is to calculate the output weight matrix based on the known signals. It can be realized simply through linear regression methods. The ridge regression method is utilized here in order to avoid overfitting to the training data. In the testing phase, untrained signals are processed to see if they belong to the same class as those used in the training phase.

\section{Fault diagnosis results and analysis}

Considering the real-time fault diagnosis process, a certain number of data samples (defined as segment width $\mathrm{W}$ herein) are collected for analysis at regular intervals (defined as sliding step $S$ herein), as shown in Fig. 8. The configuration of $S$ and $W$ is normally done according to the targeted fault types (whether they occur fast or slowly).

In the first step of our analysis, a sample-dividing procedure is performed. Each 2000-sample $V_{\text {stk }}$ in Fig. 6 is divided into a quantity of segments by applying the sliding step $(S)$ and the segment width (W) as above mentioned. And an example of $S=10$ and $W=600$, which corresponds to a sliding step of around 1s (0.91s) and a segment width of around $1 \mathrm{~min}$ (0.91 $\mathrm{min}$ ) is utilized in this study. The values are initially such selected considering that all the concerning fault types in this study (low FSC, defective cooling, CO poisoning and aging) are relatively slow ones, in time scale of minutes or even hours [49]. Certainly, to research the influence of $S$ and $W$ on the diagnostic result, different sets of these two values should be tried in this step.

The five operating conditions are labeled as the number 1-5 correspondently, as shown in Table 2. Each segment is marked as a number (1-846) and 846 segments are obtained finally for fault classification.

In the preprocessing phase, the STFT transformation is firstly applied on each segment to obtain its time-frequency representation. Consequently, the original voltage signal is transformed into a matrix $M_{c}$ with dimensions $N_{f}$ (number of frequency channels $=65) \times N_{\mathrm{s}}$ (number of time intervals or samples $=8)$. In this step, an input weight matrix $\mathrm{W}^{\mathrm{I}}\left(\mathrm{N}_{\mathrm{n}}\right.$ $(400) \times N_{f}$ ) is created randomly with a low sparsity (0.1 with 


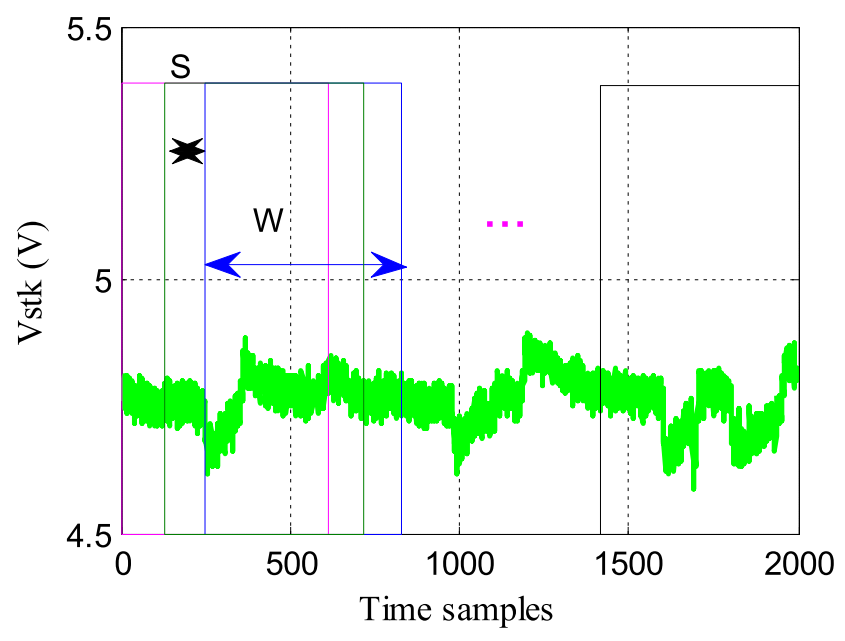

Fig. 8 - Illustration of the dividing procedure (voltage signal under FSC $=1.2$ being used as an example).

non-zero elements filled with \pm 1 ). The input data finally injected in the RC network is thus an $N_{n} \times N_{s}(400 \times 8)$ matrix $M_{u}$ (see Fig. 9, line 1). The activation of $W^{I}$ on the STFT representation can be interpreted as a random contribution of some selected frequency components at some selected time [50].

The constructed input matrix $M_{\mathrm{u}}$ is further converted into a one dimensional signal, $u(t)$, by simply unfolding horizontally each successive column and sequentially arranging them. A time trace is thus formed and injected into the dynamical system. The dynamic function as described in equation (3), performs a nonlinear transformation of the input time trace $u(t)$ into a set of reservoir states $x(t)$. The reservoir states of $R C$, $x(t)$, are further collected into a big matrix $A$, represented in a two dimensional way. Each element corresponds to the spatiotemporal response of the node amplitudes. The readout process consisting of a matrix product $A \times W^{R}=B$, with $B$ the target matrix formed by all the targets in the training dataset, as illustrated in Fig. 9.

\section{Offline training}

For offline training, a leave-one-out cross validation procedure is applied to calculate the output weight matrix and to evaluate initially the algorithm performance. Meanwhile the RC parameters above-mentioned are also optimized during this process.

Table 2 - Labelling of different operating conditions.

\begin{tabular}{llc} 
Label & Operating condition & Segment number \\
\hline 1 & Normal & $1-141$ \\
& Normal $(2 \mathrm{Nl} / \mathrm{min})$ & $283-423$ \\
2 & FSC $=1.2$ & $142-282$ \\
3 & FRW $=0.5 \mathrm{Nl} / \mathrm{min}$ & $424-564$ \\
4 & CO poisoning & $565-705$ \\
5 & Natural degradation & $705-846$ \\
\hline
\end{tabular}

The objective of the training procedure is to calculate the output weight matrix $\mathrm{W}^{\mathrm{R}}$, in order to satisfy the following condition:

$\mathrm{A} \times \mathrm{W}^{\mathrm{R}}=\mathrm{B}$

where $B$ is the target matrix formed by all the targets in the training subset. To solve this problem, multiple methods exist. In order to avoid overfitting to the training data, some Gaussian noises can be added into the training data or the ridge regression method can be applied. Herein, the second way is adopted, which introduces an additional penalty term. The penalty term serves the purpose of balancing the error on the training dataset and the weight norm [35]. The calculation of $\mathrm{W}^{\mathrm{R}}$ by ridge regression is expressed as:

$\mathrm{W}_{\text {opt }}^{\mathrm{R}}=\operatorname{argmin}_{\mathrm{W}}\left\|A \mathrm{~W}^{\mathrm{R}}-\mathrm{B}\right\|^{2}+\lambda\|\mathrm{W}\|^{2}$

where $\|\cdot\|$ is the Euclidean norm, and $\lambda$ is a regularization parameter that controls the strength of the penalty term. When $\lambda=0$, the expression becomes actually the linear regression. The term $\lambda\|W\|^{2}(\lambda>0)$ is dedicated to keep the output weights as small as possible while minimizing the training error. It should be underlined that when using ridge regression for a specific task, $\lambda$ should be optimized firstly. The calculation of the output weight matrix can be further converted as follows [50]:

$W^{R}=\left(A A^{T}+\lambda I\right)^{-1} A^{T} B$

During the leave-one-out cross validation procedure, an excellent classification rate of $99.88 \%$ has been obtained based on the offline training dataset (under the parameter set $\beta=0.8$, $\left.\phi_{0}=0.01 \pi, \lambda=5 e-5, \rho=8\right)$. Only one error of the 846 segments is obtained, which belong to the defective cooling condition and diagnosed as normal state.

The influence of principle RC parameters during this process, including the non-linearity gain $\beta$, the offset phase $\phi_{0}$, relative input scaling factor $\rho$ and the regression parameter $\lambda$, is emphasized in Section 4.3.

\section{New dataset test}

Apart from one dataset for offline training, another new dataset obtained under the same configuration of operating conditions is utilized for test. The diagnostic results are listed in Table 3. As we can see, the worst classification rate obtained is $92.435 \%$. It can be observed that all the wrongly classified segments belong to F3, i.e. the defective cooling. They are diagnosed as "Normal". This could be reasonably explained by a detailed observation of the shapes of the two voltage signals under the F3 condition (low FRW, in yellow) and the F1 condition $(2 \mathrm{Nl} / \mathrm{min}$, in purple). It can be found that they evolve with time in a quite similar way (see Table 4).

\section{Influence of RC parameters}

As mentioned in "Description of the test bench" Section, key parameters of RC method need to be optimized in order to obtain a good performance. They are namely the non-linearity gain $\beta$, the offset phase $\phi_{0}$, the relative input scaling factor $\rho$ and the regression parameter $\lambda$. The selection of the parameters is done in such a way that the range of each parameter is 


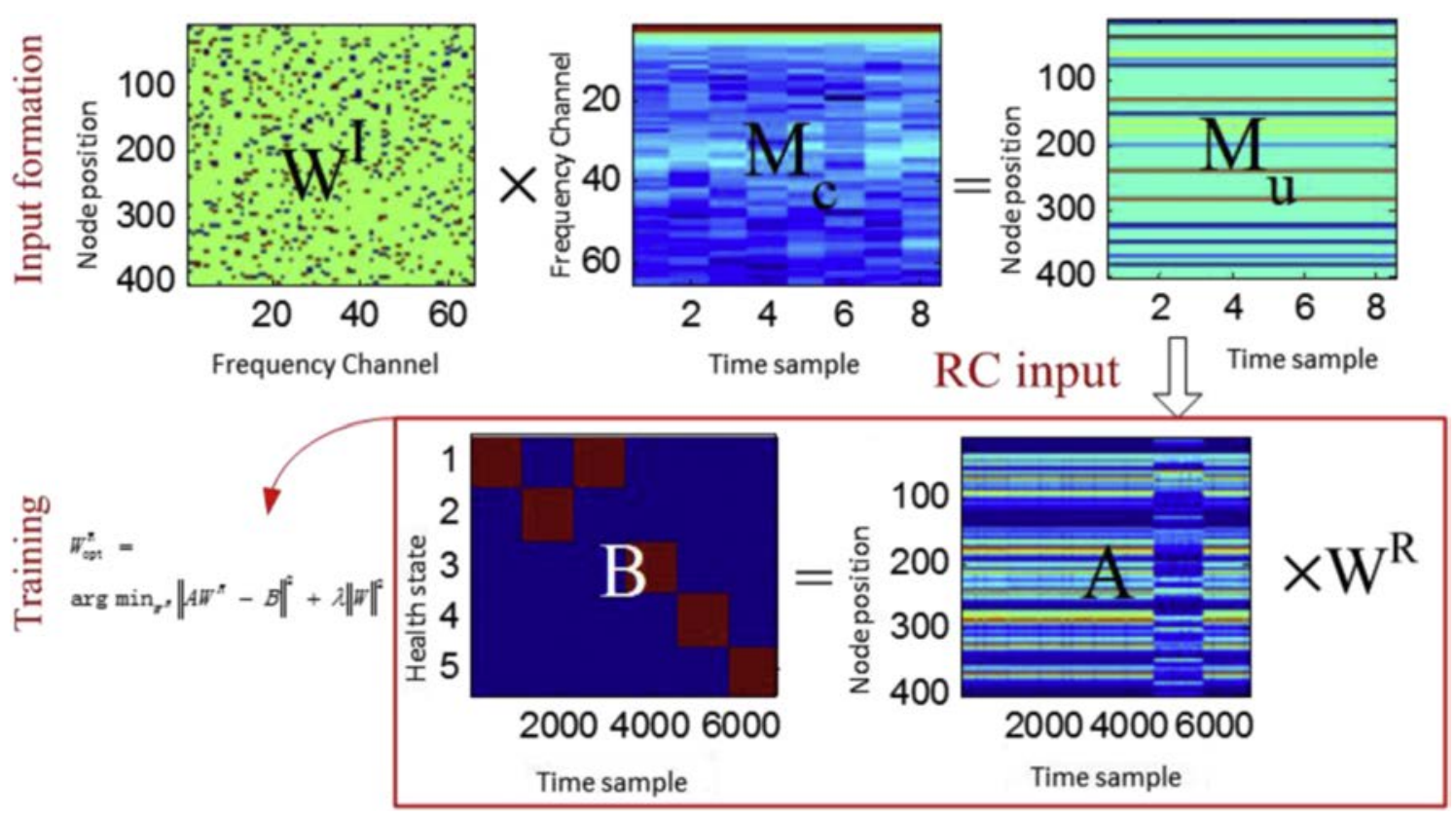

Fig. 9 - Illustration of the RC method for fault classification.

Table 3 - Online diagnostic results.

\begin{tabular}{llccccc}
\hline & & \multicolumn{5}{c}{ Diagnosed class } \\
\cline { 3 - 7 } & & F1 (normal) & F2 (low FSC) & F3 (low FRW) & F4 (CO poisoning) & F5 (severe aging) \\
\hline \multirow{2}{*}{ Actual class } & F1 (Normal) & $100 \%$ & 0 & 0 & 0 & 0 \\
& F2 & 0 & $100 \%$ & 0 & 0 & 0 \\
\\
F3 & $7.565 \%$ & 0 & $92.435 \%$ & 0 & 0 \\
& F4 & 0 & 0 & 0 & 0 & $100 \%$ \\
F5 & 0 & 0 & 0 & 0 & $100 \%$ \\
\hline
\end{tabular}

The bold is here to underline the error obtained.

Table 4 - Parameter configuration (1824 combinations of 4 parameters).

\begin{tabular}{llllll}
$\lambda$ & 0.0001 & & & 0.00001 & \\
$\rho$ & 1 & 5 & 10 & 1 & 5 \\
$\beta$ & $0.1: 0.05: 1$ & $0.1: 0.05: 1$ & $0.1: 0.05: 1$ & $0.1: 0.05: 1$ & $0.1: 0.05: 1$ \\
$\phi_{0}(/ \pi)$ & $0.01: 0.1: 1.51$ & $0.01: 0.1: 1.51$ & $0.01: 0.1: 1.51$ & $0.01: 0.1: 1.51$ & $0.01: 0.1: 1.51$ \\
\hline
\end{tabular}

given by expertise knowledge and then an exhaustive search is done to find the optimal value of each parameter corresponding to the least error rate.

The two parameters related to the non-linearity and dynamic properties- $\beta$ and $\phi_{0}$ are scanned with high precision within the given range. An example of the $\left(\beta, \phi_{0}\right)$ scanning plan with the fixed parameter $\lambda=0.00001$ and $\rho=10$ is demonstrated in Fig. 10. It can be seen that when $\beta$ is sufficiently large $(\beta>0.3)$, low classification error rates can be generally obtained. With adjustment of $\phi_{0}$, the lowest classification error rate can even reach $0.47 \%$ (e.g. $\beta=0.8$ and $\phi_{0}=0.01 \pi$ ). It can also be observed that the RC performance is quite robust versus the parameters as long as they are within a certain range, since a number of combinations can give satisfactory results.

A further research on the influences of $\rho$ and $\lambda$ is performed. Fig. 11 (a) and (b) are provided to show the dependences of the RC performance on these parameters. In the two figures, $\beta=0.8$ and $\phi_{0}=0.01 \pi$ is adopted.
It can be observed in Fig. 11 (a) that generally as $\rho$ increases, better performance can be obtained and $\rho=8$ gives the best performance. Without loss of generality, a suggested range of $\rho$ is given here: $4<\rho<10$. From the observation of Fig. 11 (b), a reasonable of choice of $\lambda$ here would be: $3 \times 10^{-5} \leq \lambda \leq 5 \times 10^{-5}$, which corresponds to a classification error rate of $0.12 \%$.

Based on the above analysis, it can be concluded that: firstly, the RC performance doesn't depend so critically on the parameters as long as they are kept within a certain range. This also explains why a number of combinations of the parameters can give the same best performance. Secondly, to perform a practical task, the mentioned "a certain range" should be specified. Herein, an (quasi) exhaustive search is applied to find the optimal parameters. However, once the parameters are determined, no iteration of the procedure is needed [41].

The values of each parameter utilized in this work are set as follows: $\beta=0.8, \phi_{0}=0.01 \pi, \rho=8, \lambda=5 e-5$. One point should be underlined is that the random generated input weight 


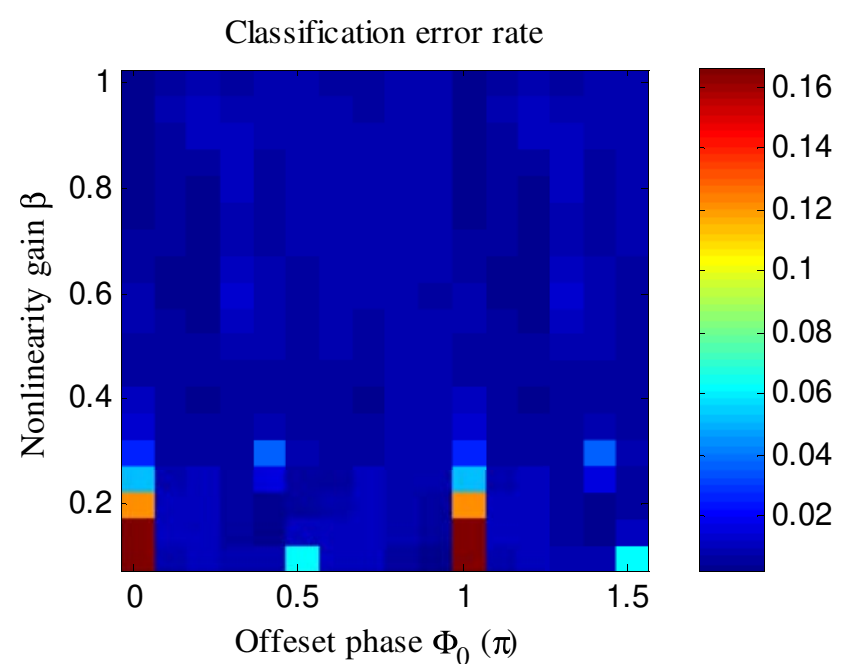

Fig. 10 - Classification error rate with two scanned parameters $\beta$ and $\phi_{0}(\lambda=0.00001, \rho=10)$.

matrix $\mathrm{W}^{\mathrm{I}}$ also has non-negligible influences on the RC performance. In the above analysis, each procedure under a new parameter set has been repeated 10 times to fix the optimal $\mathrm{W}^{\mathrm{I}}$ which corresponds to the lowest classification error rate.

\section{Influence of the learning database}

During the training procedure as indicated in "Four degrading health states" Section, 846 segments are obtained based on the 6 operating signals. Each segment goes further through a STFT preprocess to be transferred into a time-frequency $2 \mathrm{D}$ representation and corresponds to 8 time samples, as illustrated in Fig. 9. The final RC matrix A used for offline training has a dimension of 400 (number of nodes) $\times 6768$ (number of time samples), where the 6768 samples are arranged one by one in a certain order.

In this part, the aim is to analyze the influence of the construction of the learning database on the classification results. The Reservoir Computing is a tool in the field of artificial intelligence, and requires a learning phase to calibrate the synaptic weights. This learning phase involves a dedicated

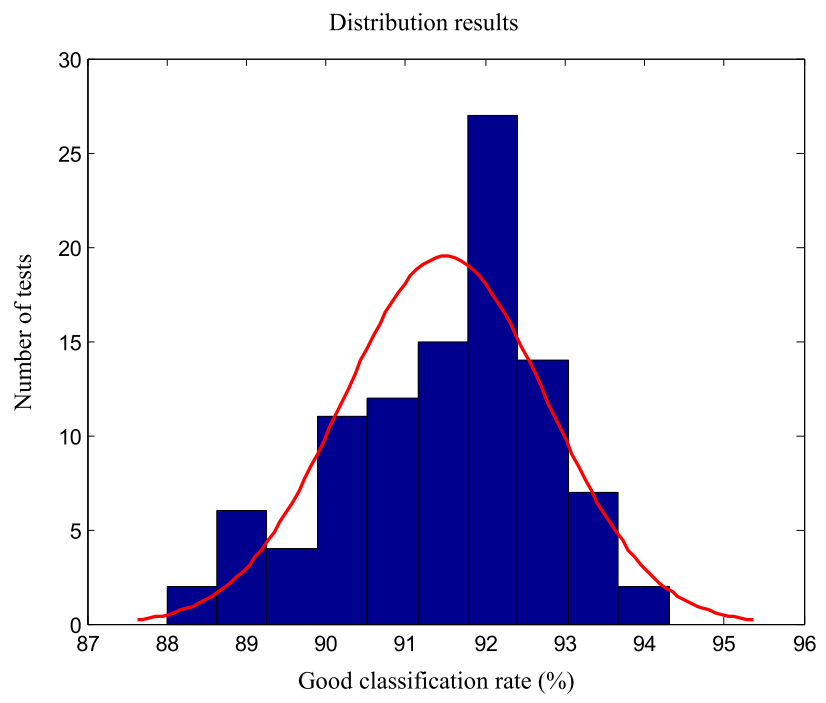

Fig. 12 - Histogram of the results (in blue) and the associated distribution fit (in red) (Study 1). (For interpretation of the references to colour in this figure legend, the reader is referred to the web version of this article.)

database for this task, and it is interesting to define how the construction of the training base will influence the results. Therefore, to determine these effects, classification rate given at the end of each study corresponds to the worst case among the results, meaning that the simulation leading to the lowest value the good classification rate is reported. In light of this, the study realized in this section try to answer the following question: Is there a difference of the classification result if only a part of the learning database is used instead of the complete one (both databases remain in order)?

A statistical tool called "P-value", which is used most often for hypothesis test in scientific research, is utilized herein. The general idea of the hypothesis test involves three steps: First, making an initial hypothesis $H_{0}$, which is also called the null hypothesis; second, collecting the database; third, based on the available data, deciding whether to accept or reject $\mathrm{H}_{0}$. In the latter case, the alternative hypothesis $\mathrm{H}_{\mathrm{a}}$ which is the opposite of the null hypothesis is accepted.
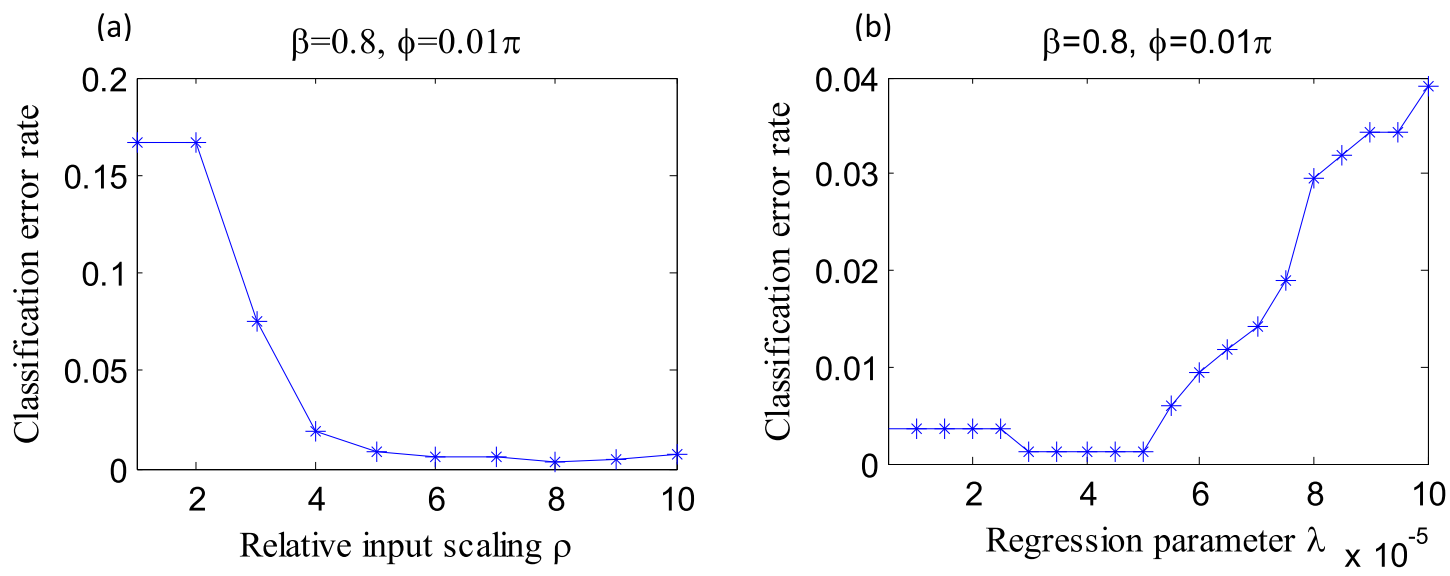

Fig. 11 - Dependence of the RC performance on (a) $\rho(\lambda=0.00001)$ and (b) $\lambda(\rho=8)$. 
A $P$-value is ultimately used to determine the probability of retaining $H_{0}$. If the $P$-value is less than (or equal to) $\alpha$, i.e. the significance level, $\mathrm{H}_{\mathrm{O}}$ is rejected. It means that the data under study gives reasonable evidence to support the alternative hypothesis. Otherwise, the initial hypothesis is retained. The choice of significant level $\alpha$ at which $\mathrm{H}_{0}$ is rejected is arbitrary and it has conventionally three levels-0.01, 0.05 and 0.1 .

Specifically, the four steps to reject or not $H_{0}$ using the $P$ value approach are [51]:

i. Specifying the null and alternative hypothesis $\left(\mathrm{H}_{0}\right.$ and $\left.\mathrm{H}_{\mathrm{a}}\right)$;

ii. Using available data and assuming that the null hypothesis is true, calculating the statistic test value. To perform hypothesis analysis for the population mean $\mu$, the value $t^{*}=\frac{\bar{x}-\mu}{s / \sqrt{n}}$ is used, which follows a t-distribution with $n-1$ degree of freedom.

iii. Using the above data, calculating the P-value corresponding to: "If the null hypothesis is true, what is the probability of observing a test statistic corresponding to the alternative hypothesis?" iv. Defining the significance level $\alpha$, compare the $P$-value to $\alpha$. If the $P$-value is less than (or equal to) $\alpha$, the null hypothesis is rejected in favor of the alternative hypothesis. If the $P$-value is greater than $\alpha$, the null hypothesis is accepted.

Study 1 of the learning database

The objective herein is to compare the simulation results obtained based on a reduced learning database with the results presented in "Four degrading health states" Section. Remind than the previous worst classification rate is $92.435 \%$, so the $\mu$ value of $\mathrm{H}_{0}$ is set as 92.43 .

To compare both situations, 100 simulations are performed by taking a smaller database, consisting of $50 \%$ of the previous database. These data are selected randomly throughout the learning database, but remain in order. Since we are interested to see whether the worst classification performance based on the reduced database is less than $92.43 \%$, and not better, a left-tailed t-test is conducted. More specifically, the

\begin{tabular}{|c|c|c|c|c|c|c|c|c|c|c|c|}
\hline $\begin{array}{r}\text { cum. prob } \\
\text { one-tail } \\
\text { two-tails }\end{array}$ & $\begin{array}{r}t_{.50} \\
0.50 \\
1.00\end{array}$ & $\begin{array}{r}t_{.75} \\
0.25 \\
0.50\end{array}$ & $\begin{array}{r}t_{.80} \\
0.20 \\
0.40\end{array}$ & $\begin{array}{r}t_{.85} \\
0.15 \\
0.30\end{array}$ & $\begin{array}{r}t_{.90} \\
0.10 \\
0.20\end{array}$ & $\begin{array}{r}t_{.95} \\
0.05 \\
0.10\end{array}$ & $\begin{array}{r}t_{.975} \\
0.025 \\
0.05\end{array}$ & $\begin{array}{r}t_{.99} \\
0.01 \\
0.02 \\
\end{array}$ & $\begin{array}{r}t_{\text {.995 }} \\
0.005 \\
0.01\end{array}$ & $\begin{array}{r}t_{\text {.999 }} \\
0.001 \\
0.002 \\
\end{array}$ & $\begin{array}{c}t_{.9995} \\
0.0005 \\
0.001 \\
\end{array}$ \\
\hline df & & & & & & & & & & & \\
\hline 1 & 0.000 & 1.000 & 1.376 & 1.963 & 3.078 & 6.314 & 12.71 & 31.82 & 63.66 & 318.31 & 636.62 \\
\hline 2 & 0.000 & 0.816 & 1.061 & 1.386 & 1.886 & 2.920 & 4.303 & 6.965 & 9.925 & 22.327 & 31.599 \\
\hline 3 & 0.000 & 0.765 & 0.978 & 1.250 & 1.638 & 2.353 & 3.182 & 4.541 & 5.841 & 10.215 & 12.924 \\
\hline 4 & 0.000 & 0.741 & 0.941 & 1.190 & 1.533 & 2.132 & 2.776 & 3.747 & 4.604 & 7.173 & 8.610 \\
\hline 5 & 0.000 & 0.727 & 0.920 & 1.156 & 1.476 & 2.015 & 2.571 & 3.365 & 4.032 & 5.893 & 6.869 \\
\hline 6 & 0.000 & 0.718 & 0.906 & 1.134 & 1.440 & 1.943 & 2.447 & 3.143 & 3.707 & 5.208 & 5.959 \\
\hline 7 & 0.000 & 0.711 & 0.896 & 1.119 & 1.415 & 1.895 & 2.365 & 2.998 & 3.499 & 4.785 & 5.408 \\
\hline & 0.000 & 0.706 & 0.889 & 1.108 & 1.397 & 1.860 & 2.306 & 2.896 & 3,355 & 4.501 & 5.041 \\
\hline 9 & 0.000 & O.ROS & 0.000 & 1.100 & 1.000 & 1.000 & 2.202 & 2.0217 & 3.250 & 4.297 & 4.781 \\
\hline 10 & 0.000 & 0.700 & 0.879 & 1.093 & 1.372 & 1.812 & 2.228 & 2.764 & 3.169 & 4.144 & 4.587 \\
\hline 11 & 0.000 & 0.697 & 0.876 & 1.088 & 1.363 & 1.796 & 2.201 & 2.718 & 3.106 & 4.025 & 4.437 \\
\hline 12 & 0.000 & 0.695 & 0.873 & 1.083 & 1.356 & 1.782 & 2.179 & 2.681 & 3.055 & 3.930 & 4.318 \\
\hline 13 & 0.000 & 0.694 & 0.870 & 1.079 & 1.350 & 1.771 & 2.160 & 2.650 & 3.012 & 3.852 & 4.221 \\
\hline 14 & 0.000 & 0.692 & 0.868 & 1.076 & 1.345 & 1.761 & 2.145 & 2.624 & 2.977 & 3.787 & 4.140 \\
\hline 15 & 0.000 & 0.691 & 0.866 & 1.074 & 1.341 & 1.753 & 2.131 & 2.602 & 2.947 & 3.733 & 4.073 \\
\hline 16 & 0.000 & 0.690 & 0.865 & 1.071 & 1.337 & 1.746 & 2.120 & 2.583 & 2.921 & 3.686 & 4.015 \\
\hline 17 & 0.000 & 0.689 & 0.863 & 1.069 & 1.333 & 1.740 & 2.110 & 2.567 & 2.898 & 3.646 & 3.965 \\
\hline 18 & 0.000 & 0.688 & 0.862 & 1.067 & 1.330 & 1.734 & 2.101 & 2.552 & 2.878 & 3.610 & 3.922 \\
\hline 19 & 0.000 & 0.688 & 0.861 & 1.066 & 1.328 & 1.729 & 2.093 & 2.539 & 2.861 & 3.579 & 3.883 \\
\hline 20 & 0.000 & 0.687 & 0.860 & 1.064 & 1.325 & 1.725 & 2.086 & 2.528 & 2.845 & 3.552 & 3.850 \\
\hline 21 & 0.000 & 0.686 & 0.859 & 1.063 & 1.323 & 1.721 & 2.080 & 2.518 & 2.831 & 3.527 & 3.819 \\
\hline 22 & 0.000 & 0.686 & 0.858 & 1.061 & 1.321 & 1.717 & 2.074 & 2.508 & 2.819 & 3.505 & 3.792 \\
\hline 23 & 0.000 & 0.685 & 0.858 & 1.060 & 1.319 & 1.714 & 2.069 & 2.500 & 2.807 & 3.485 & 3.768 \\
\hline 24 & 0.000 & 0.685 & 0.857 & 1.059 & 1.318 & 1.711 & 2.064 & 2.492 & 2.797 & 3.467 & 3.745 \\
\hline 25 & 0.000 & 0.684 & 0.856 & 1.058 & 1.316 & 1.708 & 2.060 & 2.485 & 2.787 & 3.450 & 3.725 \\
\hline 26 & 0.000 & 0.684 & 0.856 & 1.058 & 1.315 & 1.706 & 2.056 & 2.479 & 2.779 & 3.435 & 3.707 \\
\hline 27 & 0.000 & 0.684 & 0.855 & 1.057 & 1.314 & 1.703 & 2.052 & 2.473 & 2.771 & 3.421 & 3.690 \\
\hline 28 & 0.000 & 0.683 & 0.855 & 1.056 & 1.313 & 1.701 & 2.048 & 2.467 & 2.763 & 3.408 & 3.674 \\
\hline 29 & 0.000 & 0.683 & 0.854 & 1.055 & 1.311 & 1.699 & 2.045 & 2.462 & 2.756 & 3.396 & 3.659 \\
\hline 30 & 0.000 & 0.683 & 0.854 & 1.055 & 1.310 & 1.697 & 2.042 & 2.457 & 2.750 & 3.385 & 3.646 \\
\hline 40 & 0.000 & 0.681 & 0.851 & 1.050 & 1.303 & 1.684 & 2.021 & 2.423 & 2.704 & 3.307 & 3.551 \\
\hline 60 & 0.000 & 0.679 & 0.848 & 1.045 & 1.296 & 1.671 & 2.000 & 2.390 & 2.660 & 3.232 & 3.460 \\
\hline 80 & 0.000 & 0.678 & 0.846 & 1.043 & 1.292 & 1.664 & 1.990 & 2.374 & 2.639 & 3.195 & 3.416 \\
\hline 100 & 0.000 & 0.677 & 0.845 & 1.042 & 1.290 & 1.660 & 1.984 & 2.364 & 2.626 & 3.174 & 3.390 \\
\hline 1000 & 0.000 & 0.675 & 0.842 & 1.037 & 1.282 & 1.646 & 1.962 & 2.330 & 2.581 & 3.098 & 3.300 \\
\hline \multirow[t]{3}{*}{$z$} & 0.000 & 0.674 & 0.842 & 1.036 & 1.282 & 1.645 & 1.960 & 2.326 & 2.576 & 3.090 & 3.291 \\
\hline & $0 \%$ & $50 \%$ & $60 \%$ & $70 \%$ & $80 \%$ & $90 \%$ & $95 \%$ & $98 \%$ & $99 \%$ & $99.8 \%$ & $99.9 \%$ \\
\hline & \multicolumn{11}{|c|}{ nce Level } \\
\hline
\end{tabular}

Fig. 13 - Student table (study 1). 
null hypothesis is set as: $H_{0}: \mu=92.43$ versus the alternative hypothesis $H_{\mathrm{a}}: \mu<92.43$. The distribution of the 100 simulation results, corresponding to the initial population, are shown in Fig. 12. This initial population corresponds to the hypothesis $H_{0}: \mu=92.43$.

Basically, the aim here is to compare another set of simulation (the tested base) to the initial population in order to analyze the influence between both treatments. Firstly, the $t^{*}$ value has to be calculated. This value is obtained using the formula $t^{*}=\frac{\bar{x}-\mu}{s / \sqrt{n}}$, with $\bar{x}$ the average of the samples to analyze (in our case, the tested database contains 10 random samples), $\mu$ the average at which the samples are compared, $s$ the standard deviation of the sample and $n$ is the number of samples processed, i.e. $n=10$. The principle herein is to look at the value $t^{*}$ obtained for $n-1$ degrees of freedom. In this case, our value $t^{*}=-3.82$. In order to calculate the P-value, the student table or (t-table) is introduced as below. It gives the probability value $(P)$ either for one-tailed or two-two tailed test given the number of the degrees of freedom and the $t^{*}$ value (in absolute form) The aim is then to compare the obtained Pvalue with a reference value of $\alpha, 0.05$ here, a value which is often used by default in the statistic tests. In our case, the absolute value of $t^{*}$ is 3.82 and it is between 3.25 and 4.297, the column value of the Student table, as shown in Fig. 13. These two columns correspond to a P-value between 0.0005 and 0.001 , which is less than the significant level $\alpha=0.05$. As indicated in the descriptive part of the $P$-value test, the hypothesis $H_{0}$ is thus rejected, which indicated that the results are sensitive to the size of the learning database.

Nevertheless, these results are perfectly logical. It seems clear that the tools from the field of AI requiring learning are sensitive to the size of the database. However, even if this point is known to all, it remains difficult to quantify. This study let to highlight the fact that from a statistical point of view, the changes in the learning base are similar to apply a totally different treatment to the RC. This don't invalidate the study results, but it means that the way to build the learning base has importance. Upon completion of this statistical hypothesis tests, it appears that the results obtained with the RC data on fault diagnosis are dependent on the number of samples dedicated to learning. However, the study has shown that the worst simulation case give a classification rate of $88 \%$.

\section{Conclusion and perspectives}

In this paper, a first attempt of applying a recently proposed, brain-inspired computational RC method for fault diagnosis of the PEM fuel cell stack is made. The RC method originates from recurrent neural network, however has overcome the training difficulties such as low convergence rate, high computational training cost and existence of bifurcation points. The fundamental principle is to create a reservoir containing a large number (hundreds to thousands) of interconnected nodes randomly and fixedly, and to train only the output weight matrix by a linear regression method. The feasibility and efficiency of the RC method is verified by discriminating five different health states of the stack. An excellent classification rate of $99.88 \%$ for offline training process and $92.43 \%$ for new unknown dataset test are obtained. Furthermore, the influence of a set of key parameters (the non-linearity gain $\beta$, the offset phase $\phi_{0}$, the relative input scaling factor $\rho$ and the regression parameter $\lambda$ ) is emphasized on. It is summarized that RC is not extremely critical with respect to the parameters as long as they are kept within a certain range (In our case, a suggested range is: $\beta>0.3,4<\rho<10$ and $3 \times 10^{-5} \leq \lambda \leq 5 \times 10^{-5}$ ). Furthermore, the influence of the size and arranging order of the learning database is investigated. The conclusion of the study is that the classification results are dependent on these factors. However, even in the worst case, the good classification rate reach $88 \%$.

The current work is a first-stage application of RC method for PEM fuel cell fault diagnosis. Only stack voltage signals are tackled in this paper under constant current solicitation. The undergoing work involves performing fault diagnosis based on signals obtained under both stable and dynamic processes. More operating conditions and resulted fault types are configured in the second-stage work. The advantage of RC method for solving nonlinear dynamic problems is expected to be more prominent.

Additionally, the physical implementation of the algorithm will be considered in the future work for online realization of the algorithm. This photonic implementation was already achieved in the Optics Department of FEMTO-ST, UFC, for speech recognition, by Larger et al., 2012.

\section{Acknowledgment}

Experiment data from French ANR DIAPASON 2 project (ANR PAN-H 006-04) is greatly appreciated. The work performed was done within the European D-CODE project, funded under Grant Agreement 256673 of the Fuel Cells and Hydrogen Joint Technology Initiative. The authors would like to show their sincere appreciation to the sponsors and all the partners in this project.

\section{REFERENCES}

[1] Lee C, Mérida W. Gas diffusion layer durability under steadystate and freezing conditions. J Power Sources 2007;164(1):141-53. http://dx.doi.org/10.1016/ j.jpowsour.2006.09.092.

[2] Hottinen T, Himanen O, Lund P. Performance of planar freebreathing PEMFC at temperatures below freezing. J Power Sources 2006;154(1):86-94. http://dx.doi.org/10.1016/ j.jpowsour.2005.03.195.

[3] Jang J-H, Yan W-M, Chiu H-C, Lui J-Y. Dynamic cell performance of $\mathrm{kW}$-grade proton exchange membrane fuel cell stack with dead-ended anode. Appl Energy 2015;142:108-14. http://dx.doi.org/10.1016/ j.apenergy.2014.12.073.

[4] 2012 fuel cell technologies market report. U.S Department of Energy; Oct. 2013.

[5] Mench M-M, Wang C-Y, Thynell S-T. An introduction to fuel cells and related transport phenomena. Int J Transp Phenom 2001;3:151-76.

[6] Nehrir M-H, Wang C, Shaw S-R. Fuel cells: promising devices for distributed generation. IEEE Power Energy Mag 
2006;4(1):47-53. http://dx.doi.org/10.1109/

mpae.2006.1578531.

[7] Wua Jinfeng, Yuana Xiao Zi, Martina Jonathan J, Wanga Haijiang, Zhanga Jiujun, Shena Jun, et al. A review of PEM fuel cell durability: degradation mechanisms and mitigation strategies. J Power Sources 2008;184(1):104-19. http://dx.doi.org/10.1016/j.jpowsour.2008.06.006.

[8] The fuel cell industry review 2013. Fuel cell today. Jul. 2013.

[9] Barbir F. PEM fuel cells: theory and practice. 1 edition. Amsterdam; Boston: Academic Press; 2005.

[10] Wang Y, Chen K-S, Mishler J, Cho S-C, Adroher X-C. A review of polymer electrolyte membrane fuel cells: technology, applications, and needs on fundamental research. Appl Energy 2011;88(4):981-1007. http://dx.doi.org/10.1016/ j.apenergy.2010.09.030.

[11] Yuan X, Wang H, Colinsun J, Zhang J. AC impedance technique in PEM fuel cell diagnosis-a review. Int J Hydrogen Energy 2007;32(17):4365-80. http://dx.doi.org/ 10.1016/j.ijhydene.2007.05.036.

[12] Petrone R, Zheng Z, Hissel D, Péra M, Pianese C, Sorrentino M, et al. A review on model-based diagnosis methodologies for PEMFCs. Int J Hydrogen Energy 2013;38(17):7077-91.

[13] Zheng Z, Petrone R, Péra MC, Hissel D, Becherif M, Pianese C, et al. A review on non-model based diagnosis methodologies for PEM fuel cell stacks and systems. Int J Hydrogen Energy 2013;38(21):8914-26.

[14] Bethoux O, Hilairet M, Azib T. (2009). A new on-line state ofhealth monitoring technique dedicated to PEM fuel cell. Dans 35th annual conference of IEEE industrial electronics, 2009. IECON '09, pp. 2745-2750.

[15] Fouquet N, Doulet C, Nouillant C, Dauphin-Tanguy G, OuldBouamama B. Model based PEM fuel cell state-of-health monitoring via ac impedance measurements. J Power Sources 2006;159(2):905-13.

[16] Jespersen JL, Schaltz E, Kær SK. Electrochemical characterization of a polybenzimidazole-based high temperature proton exchange membrane unit cell. J Power Sources 2009;191(2):289-96.

[17] Escobet T, Feroldi D, de Lira S, Puig V, Quevedo J, Riera J, et al. Model-based fault diagnosis in PEM fuel cell systems. J Power Sources 2009;192(1):216-23.

[18] de Lira S, Puig V, Quevedo J. (2010a). Robust LPV modelbased sensor fault diagnosis and estimation for a PEM fuel cell system. Dans 2010 conference on control and fault-tolerant systems (SysTol), pp. 819-824.

[19] Buchholz M, Eswein M, Krebs V. (2008). Modelling PEM fuel cell stacks for FDI using linear subspace identification. Dans IEEE international conference on control applications, 2008. CCA 2008, pp. 341-346.

[20] Placca L, Kouta R, Candusso D, Blachot J-F, Charon W. Analysis of PEM fuel cell experimental data using principal component analysis and multi linear regression. Int J Hydrogen Energy 2010;35(10):4582-91.

[21] Zheng Z, Péra M-C, Hissel D, Becherif M, Agbli K-S, Li Y. A double-fuzzy diagnostic methodology dedicated to online fault diagnosis of proton exchange membrane fuel cell stacks. J Power Sources 2014;271:570-81.

[22] Wasterlain S, Candusso D, Harel F, François X, Hissel D. (2010). Diagnosis of a fuel cell stack using electrochemical impedance spectroscopy and Bayesian Networks. Dans 2010 IEEE vehicle power and propulsion conference (VPPC), pp. 1-6.

[23] Chen J, Zhou B. Diagnosis of PEM fuel cell stack dynamic behaviors. J Power Sources 2008;177(1):83-95.

[24] Steiner NY, Hissel D, Moçotéguy P, Candusso D. Non intrusive diagnosis of polymer electrolyte fuel cells by wavelet packet transform. Int J Hydrogen Energy 2011;36(1):740-6.
[25] Yousfi Steiner N, Hissel D, Moçotéguy P, Candusso D. Diagnosis of polymer electrolyte fuel cells failure modes (flooding \& drying out) by neural networks modeling. Int J Hydrogen Energy 2011;36(4):3067-75. http://dx.doi.org/ 10.1016/j.ijhydene.2010.10.077.

[26] Kim J, Lee I, Tak Y, Cho BH. State-of-health diagnosis based on hamming neural network using output voltage pattern recognition for a PEM fuel cell. Int J Hydrogen Energy 2012;37(5):4280-9.

[27] Rouss V, Charon W. Multi-input and multi-output neural model of the mechanical nonlinear behaviour of a PEM fuel cell system. J Power Sources 2008;175(1):1-17. http:// dx.doi.org/10.1016/j.jpowsour.2007.09.008.

[28] Lobatoa Justo, Cañizaresa Pablo, Rodrigoa Manuel A, Linaresa José J, Piuleacb Ciprian-George, Curteanub Silvia. The neural networks based modeling of a polybenzimidazolebased polymer electrolyte membrane fuel cell: effect of temperature. J Power Sources 2009;192(1):190-4. http:// dx.doi.org/10.1016/j.jpowsour.2009.01.079.

[29] Jemei S, Hissel D, Péra M-C, Kauffmann J-M. On-board fuel cell power supply modeling on the basis of neural network methodology. J Power Sources 2003;124(2):479-86. http:// dx.doi.org/10.1016/S0378-7753(03)00799-7.

[30] Zhang L, Pan M, Quan S. Model predictive control of water management in PEMFC. J Power Sources 2008;180(1):322-9. http://dx.doi.org/10.1016/j.jpowsour.2008.01.088.

[31] El-Sharkh MY, Rahman A, Alam MS. Neural networks-based control of active and reactive power of a stand-alone PEM fuel cell power plant. J Power Sources 2004;135(1-2):89-94. http://dx.doi.org/10.1016/j.jpowsour.2004.03.071.

[32] Larger L, Soriano M-C, Brunner D, Appeltant L, Gutierrez J-M, Pesquera L, et al. Photonic information processing beyond Turing: an optoelectronic implementation of reservoir computing. Opt Express 2012;20(3):3241-9. http://dx.doi.org/ 10.1364/oe.20.003241.

[33] Lukoševičius $M$, Jaeger $H$. Reservoir computing approaches to recurrent neural network training. Comput Sci Rev 2009;3(3):127-49. http://dx.doi.org/10.1016/ j.cosrev.2009.03.005.

[34] Doya K. Bifurcations in the learning of recurrent neural networks. Proc IEEE Int Symposium Circuits Syst (ISCAS) 1992;6:2777-80. http://dx.doi.org/10.1109/iscas.1992.230622.

[35] Appeltant L, Soriano M-C, Van der Sande G, Danckaert J, Massar S, Dambre J, et al. Information processing using a single dynamical node as complex system. Nat Commun 2011;2:468. http://dx.doi.org/10.1038/ncomms1476.

[36] Brunner D, Soriano M-C, Mirasso C-R, Fischer I. Parallel photonic information processing at gigabyte per second data rates using transient states. Nat Commun 2013;4:1364.

[37] Jaeger H. The "echo state" approach to analysing and training recurrent neural networks with an erratum note. German National Research Center for Information Technology GMD Technical Reportvol. 148; 2001. p. 34.

[38] Maass W, Natschläger T, Markram H. Real-time computing without stable states: a new framework for neural computation based on perturbations. Neural Comput 2002;14(11):2531-60. http://dx.doi.org/10.1162/ 089976602760407955.

[39] Verstraeten D, Schrauwen B, D'Haene M, Stroobandt D. An experimental unification of reservoir computing methods. Neural Netw 2007;20(3):391-403. http://dx.doi.org/10.1016/ j.neunet.2007.04.003.

[40] Verstraeten D, Schrauwen B, Stroobandt D. Reservoir-based techniques for speech recognition. International joint conference on neural networks (IJCNN) 2006. pp. 1050-53. doi:10.1109/ijcnn.2006.246804.

[41] Jaeger H, Lukoševičius M, Popovici D, Siewert U. Optimization and applications of echo state networks with 
leaky-integrator neurons. Neural Netw 2007;20(3):335-52. http://dx.doi.org/10.1016/j.neunet.2007.04.016.

[45] Hissel D, Péra M-C, Candusso D, Harel F, Begot S. Characterization of polymer electrolyte fuel cells for embedded generators: test bench design and methodology. Adv Fuel Cells 2005:127-48.

[47] Li Z, Outbib R, Giurgea S, Hissel D, Li Y. Fault detection and isolation for polymer electrolyte membrane fuel cell systems by analyzing cell voltage generated space. Appl Energy 2015;148:260-72. http://dx.doi.org/10.1016/ j.apenergy.2015.03.076.

[48] Li Z, Outbib R, Giurgea S, Hissel D. Diagnosis for PEMFC systems: a data-driven approach with the capabilities of online adaptation and novel fault detection. IEEE Trans Ind Electron 2015;62(8):5164-74. http://dx.doi.org/10.1109/ tie.2015.2418324.

[49] Li Z, Outbib R, Giurgea S, Hissel D, Jemei S, Giraud A, et al. Online implementation of SVM based fault diagnosis strategy for PEMFC systems. Appl Energy 2015;164:284-93. http://dx.doi.org/10.1016/j.apenergy.2015.11.060.

[50] Verstraeten D. Reservoir Computing: computation with dynamical systems. PhD thesis. Ghent University; 2009.

[51] Motulsky H. Intuitive biostatistics: a nonmathematical guide to statistical thinking. 3rd ed. Oxford University Press; 2013. 